\title{
XMM-Newton observation of the bright Seyfert 1 galaxy, $M C G+8-11-11$
}

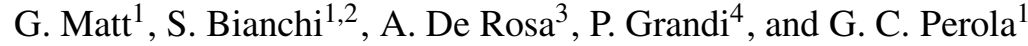 \\ 1 Dipartimento di Fisica, Università degli Studi Roma Tre, via della Vasca Navale 84, 00146 Roma, Italy \\ e-mail: matt@fis.uniroma3.it \\ 2 XMM-Newton Science Operation Center, ESAC/ESA, Apartado 50727, 28080 Madrid, Spain \\ 3 IASF - Sezione di Roma, INAF, via del Fosso del Cavaliere 100, 00113 Roma, Italy \\ ${ }^{4}$ IASF - Sezione di Bologna, INAF, via Gobetti 101, 40129 Bologna, Italy
}

Received 9 August 2005 / Accepted 1 September 2005

\section{ABSTRACT}

We report on the XMM-Newton observation of the bright Seyfert 1 galaxy, MCG+8-11-11. Data from the EPIC/p-n camera, the Reflection Gratings Spectrometers (RGS), and the Optical Monitor (OM) were analyzed. The p-n spectrum was fitted well by a power law, a spectrally unresolved $\mathrm{Fe} \mathrm{K} \alpha$ line, a Compton reflection component (whose high value, when compared to the iron line equivalent width, suggests iron underabundance), and absorption by warm material. Absorption lines are apparent in the RGS spectra, but their identification is uncertain and would require large matter velocities. The UV fluxes measured by the OM are well above the extrapolation of the X-ray spectrum, indicating the presence of a UV bump.

Key words. galaxies: active - X-rays: galaxies - galaxies: individual: MCG+8-11-11

\section{Introduction}

One of the main surprises from Chandra and especially XMM-Newton observations of Seyfert galaxies is that the iron $\mathrm{K} \alpha$ line, while confirmed to be almost ubiquitous, is often (but not always) spectrally unresolved and therefore unlikely to originate in the innermost regions of the accretion disc. While relativistically broadened lines have sometimes been confirmed (e.g. in MCG-6-30-15, Wilms et al. 2001 and Fabian et al. 2002; NGC 3516, Turner et al. 2002; MCG-5-23-16, Balestra et al. 2004), in many objects only the unresolved component is visible, the upper limits to the relativistic component being quite tight (e.g. Bianchi et al. 2004). The reason for a different behavior from source to source is at present unclear. Among the proposed explanations are disc truncation and significant ionization of the matter.

MGC+8-11-11 $(z=0.0205)$ is one of the brightest AGN in the X-ray band. It has been observed by all major X-ray satellites, with the notable exception of Chandra. ASCA (Grandi et al. 1998) and BeppoSAX (Perola et al. 2000) found that the spectrum is fitted well by a fairly standard model composed of a power law, a warm absorber, a Compton reflection component, and an iron $\mathrm{K} \alpha$ line. The moderate energy resolution and/or sensitivity of those instruments left undecided whether the line originated in the innermost regions of the accretion disc (and hence relativistically broadened) or in distant matter (and thence unresolved). One of the main goals of the $\mathrm{XMM}-$ Newton observation of this source is to solve this issue.

\section{Observation and data reduction}

MCG+8-11-11 was observed by XMM-Newton on 10 April 2004 [OBSID 0201930201] with the EPIC p-n (Strüder et al. 2001) and MOS (Turner et al. 2001) cameras, both in small window/medium filter mode, with the RGS and the OM. Data were reduced with SAS 6.0.0 adopting standard procedures, while screening for intervals of flaring particle background in the EPIC data was done consistently with the choice of the extraction radii, in order to maximise the signal-to-noise ratio, similar to what described by Piconcelli et al. (2004). As a result of this procedure, the effective time and the source extraction radius in the $p-n$ are $37.7 \mathrm{ks}$ and 40 arcsec. Patterns 0 to 4 were included in the p-n spectrum, whose count rate (14.1 counts/s) is lower than the maximum for 1 per cent pile-up (see Table 3 of Ehle et al. 2005). Effective time in the RGS is $38.2 \mathrm{ks}$.

We did not make use of MOS data in this paper for two reasons. First, the MOS are slightly piled-up, despite the small window mode adopted. And second, just because of the small window, it is very difficult to extract the background in the field-of-view, and the use of blank fields is then necessary, which may oversubtract the spectrum at low energies due to the high Galactic column to the source.

The p-n spectra were rebinned to oversample the energy resolution by a factor about 3 and, if necessary, further rebinned until at least 25 counts/bin are obtained. For the gratings spectra, only the latter criterium was adopted. Six exposures with the Optical Monitor were also available, three with the 


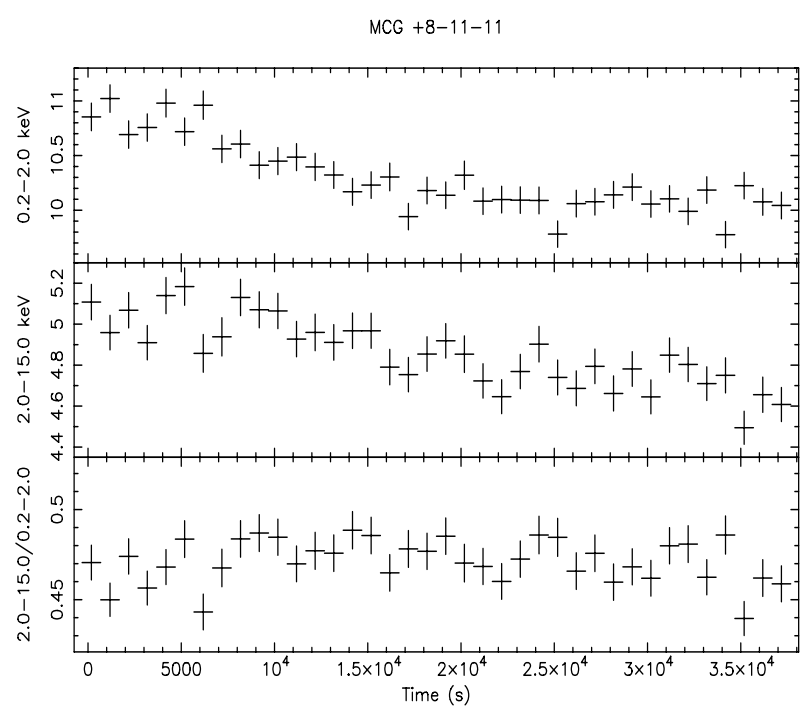

Fig. 1. The lightcurves in the $0.2-2 \mathrm{keV}$ (upper panel) and in the $2-15 \mathrm{keV}$ band (middle panel). The lower panel shows the $(2-15) /(0.2-2) \mathrm{keV}$ hardness ratio.

$U V W 1(291 \mathrm{~nm})$ and three with the $U V W 2(212 \mathrm{~nm})$ filters. In the following, all errors refer to $90 \%$ confidence level for one interesting parameter $\left(\Delta \chi^{2}=2.71\right)$.

\section{Results}

\subsection{Temporal analysis}

During the observation, the source flux varied by about $10 \%$, with no significant spectral variations (see Fig. 1). We then divided the observation into five equally spaced intervals. After having verified that the spectral parameters in the five intervals remained constant within the errors, we searched for the presence of absorption or emission transient features, like those supposedly present in the spectra of many Seyfert galaxies (e.g. Pecháček et al. 2005 and references therein; Matt et al. 2005; Dadina et al. 2005). No significant feature was detected.

\subsection{Spectral analysis}

The lack of any significant spectral variability allowed us to use the whole observation for spectral purposes. We started fitting only the $\mathrm{p}-\mathrm{n}$ instrument in the $0.5-12 \mathrm{keV}$ energy range. The spectrum and residuals when fitting with a simple absorbed power law are shown in Fig. 2. The fit is clearly unacceptable with a $\chi^{2} /$ d.o.f. of $697.1 / 260$. An iron $\mathrm{K} \alpha$ emission line is the most obvious feature present in the residuals; an iron $\mathrm{K}$ edge and wiggles in the softer part of the spectrum are also apparent. From this and following Perola et al. (2000), we fitted the spectrum with a model composed by an absorbed power law, a Compton reflection component (model PEXRAV in XSPEC, with the inclination angle kept fixed to $30^{\circ}$ ), a Gaussian iron $\mathrm{K} \alpha$ line, and warm absorption (model ABSORI, with the temperature of the material kept fixed to $10^{6} \mathrm{~K}$ ). Metal abundances were kept fixed to cosmic ones, according to Anders \& Grevesse (1989). All these components are required by the data. Addition of the iron line, the reflection

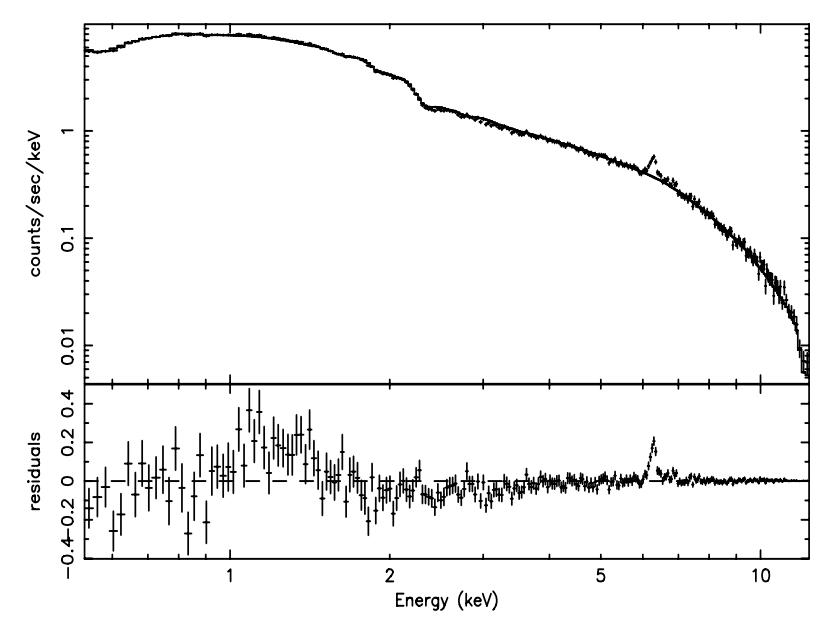

Fig. 2. Spectrum and best fit model (upper panel) and residuals (lower panel), when fitted with a simple absorbed power law.

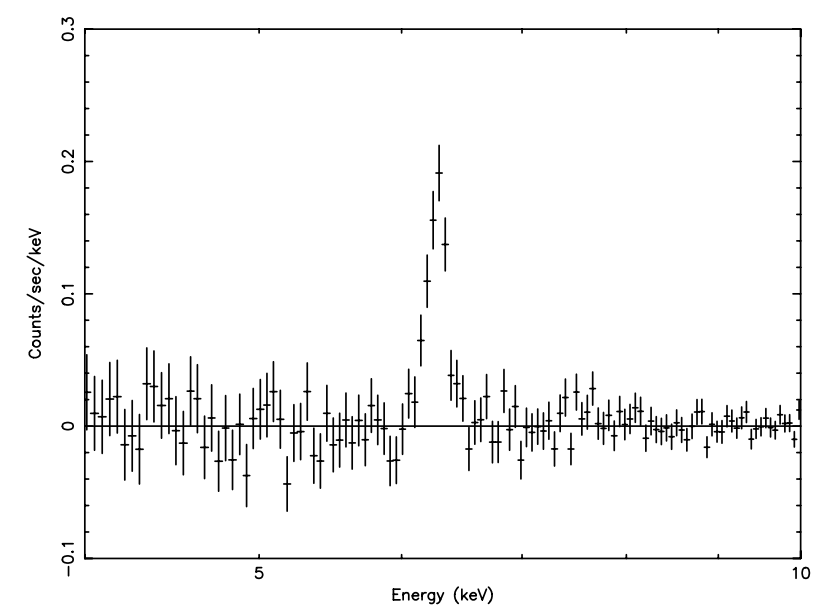

Fig. 3. Residuals around the iron line energy band. The plot has been obtained by first fitting the spectrum with the model described in Table 1, and then removing the iron line, a technique used for illustration purposes only.

component, and the warm absorber in turn gives values of $\chi^{2} /$ d.o.f. of $393.4 / 257,278.4 / 256$, and $256.8 / 254$. The final fit is perfectly acceptable. The iron line is unresolved $(\sigma<60 \mathrm{eV}$; see also Fig. 3 and therefore we decided, in the following fits, to simplify by fixing its width to $1 \mathrm{eV}$.

Inspection of the residuals showed a deficit of counts around $0.7-0.9 \mathrm{keV}$. This suggests that a single-zone warm absorption model is insufficient to describe the data. We therefore added an edge at $0.74 \mathrm{keV}$, corresponding to He-like Oxygen. The addition of this component leads to $\chi^{2} /$ d.o.f. $=241.9 / 254$.

We then added the iron $\mathrm{K} \beta$ line, the nickel $\mathrm{K} \alpha$ line, and the iron $\mathrm{K} \alpha$ Compton Shoulder (CS hereinafter). All three features are expected to go along with the iron $\mathrm{K} \alpha$ line. The first two lines were modelled with unresolved $(\sigma=1 \mathrm{eV})$ Gaussians with energies fixed at 7.06 and $7.48 \mathrm{keV}$, the CS with a Gaussian with centroid energy of $6.3 \mathrm{keV}$ and $\sigma=40 \mathrm{eV}$ (Matt 2002). The CS is marginally significant, while only upper limits are obtained for the other two lines. Finally, it is worth noting that, unlike with many other Seyfert galaxies and quasars, no soft excess is required by the data (see Fig. 4). 


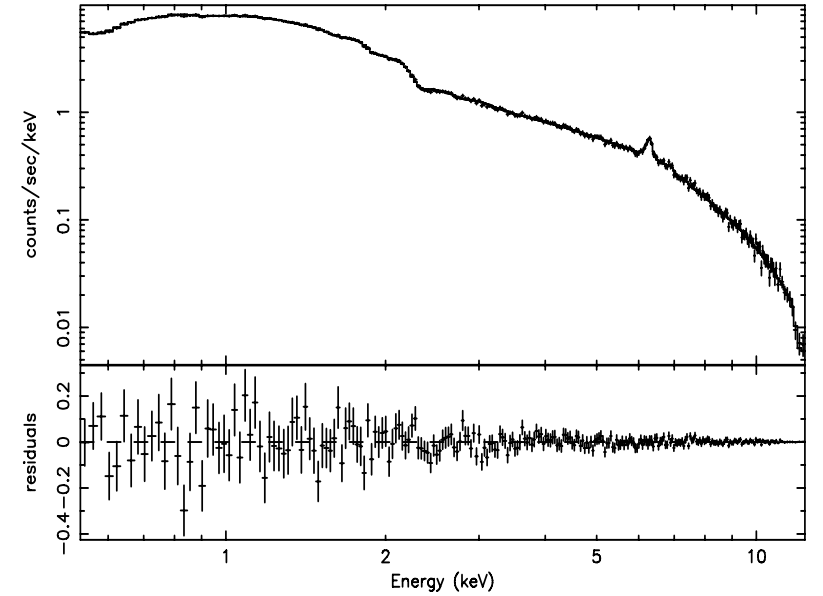

Fig. 4. Spectrum and best fit model (upper panel) and resisuals (lower panel). See Table 1 for the best fit parameters.

The best fit parameters are summarized in Table 1, while both the spectrum with the best fit model and the residuals are shown in Fig. 4. The value of the column density, $\sim 1.8 \times 10^{21} \mathrm{~cm}^{-2}$, is consistent with the Galactic one. Elvis et al. (1989) found a value of $(2.0 \pm 0.2) \times 10^{21} \mathrm{~cm}^{-2}$. Fixing the $N_{\mathrm{H}}$ to this value leads to a significantly worse, even if still perfectly acceptable, fit, i.e. $\chi^{2} /$ d.o.f. $=255.4 / 252$. The power law is of course steeper, i.e. 1.9, which in turn gives a very large value of $R$, i.e., 2.6 . The $2-10 \mathrm{keV}$ unabsorbed flux is $4.63 \times 10^{-11} \mathrm{erg} \mathrm{cm}^{-2} \mathrm{~s}^{-1}$, corresponding to a luminosity in the same band of $4.3 \times 10^{43} \mathrm{erg} \mathrm{s}^{-1}\left(H_{0}=70 \mathrm{~km} \mathrm{~s}^{-1} \mathrm{Mpc}^{-1}\right)$. As far as we know, there is no estimate of the Black Hole mass based on BLR reverberation mapping. Using the BLR radius vs the $5100 \AA$ luminosity relationship (Kaspi et al. 2000) and the width of the BLR lines, Bian \& Zhao (2003) estimate a Black hole mass of about $1.5 \times 10^{7} M_{\odot}$, implying an Eddington luminosity of about $2 \times 10^{45} \mathrm{erg} \mathrm{s}^{-1}$. Hence, $L_{\mathrm{Bol}} / L_{\mathrm{edd}}=0.02 \mathrm{k}$, where $k$ is the bolometric correction, which is about 25 for the observed X-ray luminosity according to Marconi et al. (2004). Therefore, the source would be emitting at about half the Eddington limit, which seems unlikely given the spectrum (high $\dot{m}$ sources, like NLS1 probably are, have usually much steeper spectra and prominent soft excesses). A more accurate Black Hole mass estimate is needed before drawing any conclusion in this respect.

\subsubsection{The reflection component and the emission lines}

The iron line is unresolved, suggesting an origin in matter that is more distant than the innermost regions of the accretion disc. This is similar to what has been found in many other bright, local Seyfert galaxies (e.g. Bianchi et al. 2004). The clear presence of a reflection component (the fit without this component gives a $\chi^{2} /$ d.o.f. of $378.6 / 252$ ) indicates that the line-emitting matter is Compton-thick (e.g. Matt et al. 2003). This is consistent with the admittedly loose upper limit to the CS-to-line core ratio (about 0.4 ). Natural candidates for the line emitting region are the outermost part of the disc and the "torus"
Table 1. Parameters of the best fit model.

\begin{tabular}{|c|c|}
\hline$\Gamma$ & $1.805 \pm 0.015$ \\
\hline$N_{\mathrm{H}}$ & $1.83_{-0.03}^{+0.06} \times 10^{21} \mathrm{~cm}^{-2}$ \\
\hline$R$ & $1.51_{-0.09}^{+0.13}$ \\
\hline$N_{\mathrm{H}, \text { warm }}$ & $1.1_{-0.6}^{+0.6} \times 10^{22} \mathrm{~cm}^{-2}$ \\
\hline$\xi$ & $460_{-130}^{+270} \mathrm{erg} \mathrm{cm} \mathrm{s}^{-1}$ \\
\hline$\tau_{\text {edge }}$ & $0.041_{-0.014}^{+0.018}$ \\
\hline$E(\mathrm{Fe} \mathrm{K} \alpha)$ & $6.416_{-0.004}^{+0.014} \mathrm{keV}$ \\
\hline$F(\mathrm{Fe} \mathrm{K} \alpha)$ & $4.1_{-0.6}^{+0.6} \times 10^{-5} \mathrm{ph} \mathrm{cm}^{-2} \mathrm{~s}^{-1}$ \\
\hline$E W(\mathrm{Fe} \mathrm{K} \alpha)$ & $75 \pm 15 \mathrm{eV}$ \\
\hline$F(\mathrm{Fe} \mathrm{K} \beta)$ & $4.6_{-4.5}^{+4.4} \times 10^{-6} \mathrm{ph} \mathrm{cm}^{-2} \mathrm{~s}^{-1}$ \\
\hline$E W(\mathrm{Fe} \mathrm{K} \beta)$ & $<10 \mathrm{eV}$ \\
\hline$F(\mathrm{Ni} \mathrm{K} \alpha)$ & $4.7_{-4.4}^{+4.5} \times 10^{-6} \mathrm{ph} \mathrm{cm}^{-2} \mathrm{~s}^{-1}$ \\
\hline$E W(\mathrm{Ni} \mathrm{K} \alpha)$ & $<24 \mathrm{eV}$ \\
\hline$F(\mathrm{Fe} \mathrm{K} \alpha \mathrm{CS})$ & $1.1_{-0.8}^{+0.7} \times 10^{-5} \mathrm{ph} \mathrm{cm}^{-2} \mathrm{~s}^{-1}$ \\
\hline$E W(\mathrm{Fe} \mathrm{K} \alpha \mathrm{CS})$ & $17 \pm 12 \mathrm{eV}$ \\
\hline Flux $(2-10 \mathrm{keV})$ & $4.63 \times 10^{-11} \mathrm{erg} \mathrm{cm}^{-2} \mathrm{~s}^{-1}$ \\
\hline$\chi^{2} /$ d.o.f. & $232.5 / 251$ \\
\hline
\end{tabular}

envisaged in unification models (Antonucci 1993). In the latter hypothesis, no variability of the iron line during the observation is expected; indeed, no variability is found. It should be noted, however, that if the line were to follow variations of the continuum, $10 \%$ variations would be expected, which is still consistent with the data. In the former hypothesis, the inner radius of the disc-emitting region is about 300 gravitational radii, as derived from fitting the iron line with a DISKLINE model, with inclination angle set to $30^{\circ}$ and outer radius to $10^{4}$ gravitational radii (as good statistically as that with a gaussian line).

The ratio between the $\mathrm{Fe} \mathrm{K} \beta$ and $\mathrm{K} \alpha$ lines is constrained to be less than about $20 \%$, therefore giving no useful informations on the ionization state of iron (see Molendi et al. 2003). A similar upper limit to the ratio holds for the $\mathrm{Ni}$ to $\mathrm{Fe} \mathrm{K} \alpha$ line flux ratio, providing only a very loose constraint on the Ni-to-Fe abundance, i.e. less than about 5.

The iron $\mathrm{K} \alpha$ line $E W$, about $90 \mathrm{eV}$ (including the CS), is lower than expected given the large amount of the reflection component (e.g. George \& Fabian 1991; Matt et al. 1991) ${ }^{1}$. There are two possible explanations for that.

The first explanation is that iron is underabundant. We therefore left the iron abundance (in units of the solar value) in the PEXRAV model free to vary. The best fit gives $A_{\mathrm{Fe}}=0.78$, but the improvement in the fit is not significant. In Fig. 5 we show the contour plot of $R$ and $A_{\mathrm{Fe}}$. A solution with $R \simeq 1.2$ and $A_{\mathrm{Fe}} \simeq 0.5$ is acceptable on both statistical and physical (see Matt et al. 1997) grounds.

${ }^{1}$ Fixing $R$ (i.e. the solid angle subtended by the reflecting matter in units of $2 \pi$ ) to 1 the fit is significantly worse, i.e. $\chi^{2} /$ d.o.f. $=$ $283.5 / 252$. Note also that fitting the spectrum without any warm absorption gives even higher values of $R$. 


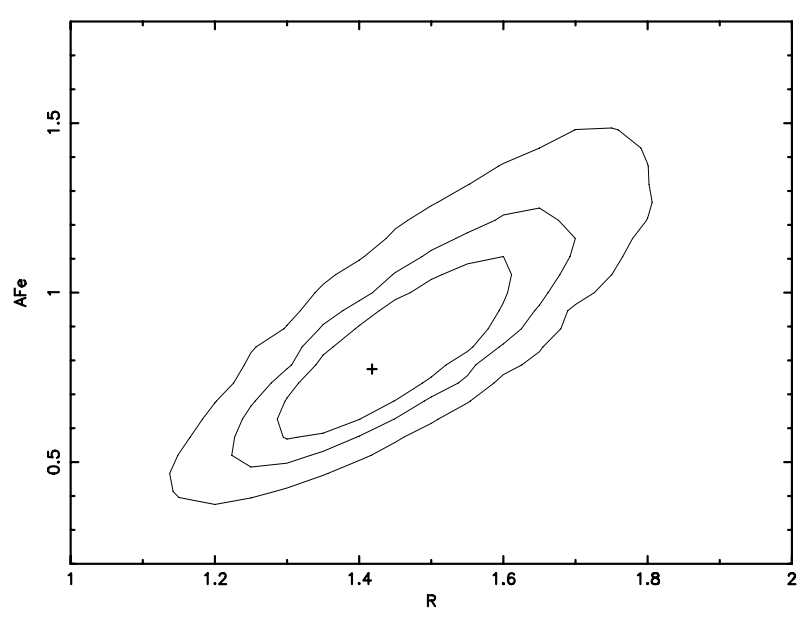

Fig. 5. $R$ and iron abundance contour plot.

Alternatively, it is concevaible that there are two distinct reflecting regions: one cold, distant, and associated with the observed iron line; the other mildly ionized and probably arising from the accretion disc. Line emission from the latter regions would then be prevented by resonant trapping (e.g. Matt et al. 1996). To test this hypothesis, we added a mildly ionized reflector with relativistic blurring (model REFSCH), with ionization parameter fixed to $400 \mathrm{erg} \mathrm{cm} \mathrm{s}^{-1}$ (as appropriate to dump line emission), inner and outer radii fixed to 6 and 1000 gravitational radii, and the inclination angle to $30^{\circ}$. The amount of cold reflection has been kept fixed to $R=0.7$, as is appropriate given the observed iron line $E W$. The fit is acceptable $\left(\chi^{2} /\right.$ d.o.f. $\left.=285.9 / 251\right)$, but significantly worse than with a single cold reflector. The best fit value for $R, 0.25_{-0.02}^{+0.04}$ is much lower than expected in a standard disc-corona scenario (i.e. $R=1$ ). We then allowed the inner radius of the disc to vary, but no better fit was found.

In summary, iron underabundance seems to be preferred both on statistical and physical grounds.

\subsubsection{The warm absorber}

A single-zone warm absorber (modelled with ABSORI in XSPEC) gives an acceptable fit, but some residuals remain, which are cured well by the addition of an $\mathrm{O}$ VII absorption edge. This suggests the presence of either two different absorbing regions, but a fit with two ABSORI models provides a slightly worse fit, or of a complex absorber. We also checked that the residuals are not due to keeping the temperature of the absorber fixed to 1 million degrees. In fact, when leaving the temperature free to vary, only a slight improvement in the fit is found. The fit is, in any case, worse $\left(\chi^{2} /\right.$ d.o.f. $\left.=249.7 / 252\right)$ than with the temperature fixed and the absorption edge. We also searched for a possible inflow or outflow of the warm absorber. The result is quite disappointing, as the upper limit to the outflow velocity is about $3 \times 10^{4} \mathrm{~km} \mathrm{~s}^{-1}$.

Of course, it is also possible that there is only one absorbing region, but that the model we adopted is too simple. The absorbing region may well be stratified, resulting in a gradient of ionization parameters (see e.g. Różańska et al. 2004 for
Table 2. Absorption lines detected in the RGS spectra with a flux percentage error less than $50 \%$. Both observed and rest-frame energies are given.

\begin{tabular}{cccc}
\hline \hline$E_{\text {obs }}(\mathrm{eV})$ & $E_{\text {r.f. }}(\mathrm{eV})$ & $F\left(\mathrm{ph} \mathrm{cm}^{-2} \mathrm{~s}^{-1}\right)$ & $E W(\mathrm{eV})$ \\
\hline $526 \pm 1$ & $537 \pm 1$ & $-1.50_{-0.27}^{+0.39} \times 10^{-4}$ & -3.9 \\
$586 \pm 1$ & $598 \pm 1$ & $-1.24_{-0.54}^{+0.30} \times 10^{-4}$ & -4.0 \\
$627 \pm 1$ & $640 \pm 1$ & $-0.66_{-0.22}^{+0.24} \times 10^{-4}$ & -2.4 \\
$654 \pm 1$ & $667 \pm 1$ & $-0.56_{-0.20}^{+0.21} \times 10^{-4}$ & -2.1 \\
$999 \pm 1$ & $1020 \pm 5$ & $-0.41_{-0.21}^{+0.21} \times 10^{-4}$ & -3.4 \\
\hline
\end{tabular}

a constant pressure model). A detailed modeling of the warm absorption in MCG+8-11-11 is beyond the scope of this paper; however, a longer exposure observation, able to fully exploit the energy resolution of the gratings, would be required to achieve this aim.

Given the brightness of the source, the RGS instruments can still provide some information on the warm matter, especially on narrow absorption or emission lines. We fitted the RGS data with the same model used for the p-n, with all parameters fixed to the best fit values. Inspection of the residuals (see Fig. 6) shows a number of possible absorption lines. We checked that these lines are still present when the continuum is fitted locally, e.g. by power laws. Note also that for the two of them for which both gratings were available, i.e. those at 640 and $667 \mathrm{eV}$, the lines are visible in both instruments. In Table 2 we list the five lines we found with a flux percentage error less than $50 \%$. The identification of these lines is not obvious. None of them are among the possible instrumental lines discussed by Ravasio et al. (2005). Only the $667 \mathrm{eV}$ and the 1020 lines have energies corresponding to known and relatively significant transitions (see e.g. Bianchi et al. 2005 for a list of relevant lines), i.e. the $\mathrm{O}$ VII $\mathrm{K} \beta$ and the $\mathrm{Ne} \mathrm{x} \mathrm{K} \alpha$ lines. The absence of the corresponding $\mathrm{K} \alpha$ line makes, however, the former identification quite unlikely. It must also be noted that the observed line energy, $0.654 \pm 0.001 \mathrm{keV}$, is close to that of the $\mathrm{O}$ VIII $\mathrm{K} \alpha$ line, suggesting a possible origin in our own Galaxy or the local group. We were also unable to find a single velocity able to provide a realistic identification even for only two lines. Moreover, after tentatively identifying the lines with the closest Oxygen lines, velocities should be about several thousands $\mathrm{km} / \mathrm{s}$ at least, so much larger than usually observed in warm absorbers (e.g. Kaspi et al. 2002), even if not dissimilar to those observed in a few high luminosity sources (e.g. Hasinger et al. 2002; Chartas et al. 2002; Pounds et al. 2003a,b). No further information on the velocity structure can be derived from the absorption edges, which in the RGS are only an upper limit. Clearly, a much longer observation is needed to confirm the presence of these lines and to search for more lines and, then, for possible systems at the same velocity.

\subsection{The ultraviolet flux}

The UV fluxes of the OM observations were obtained by simply converting the count rates with the method 3 described in 

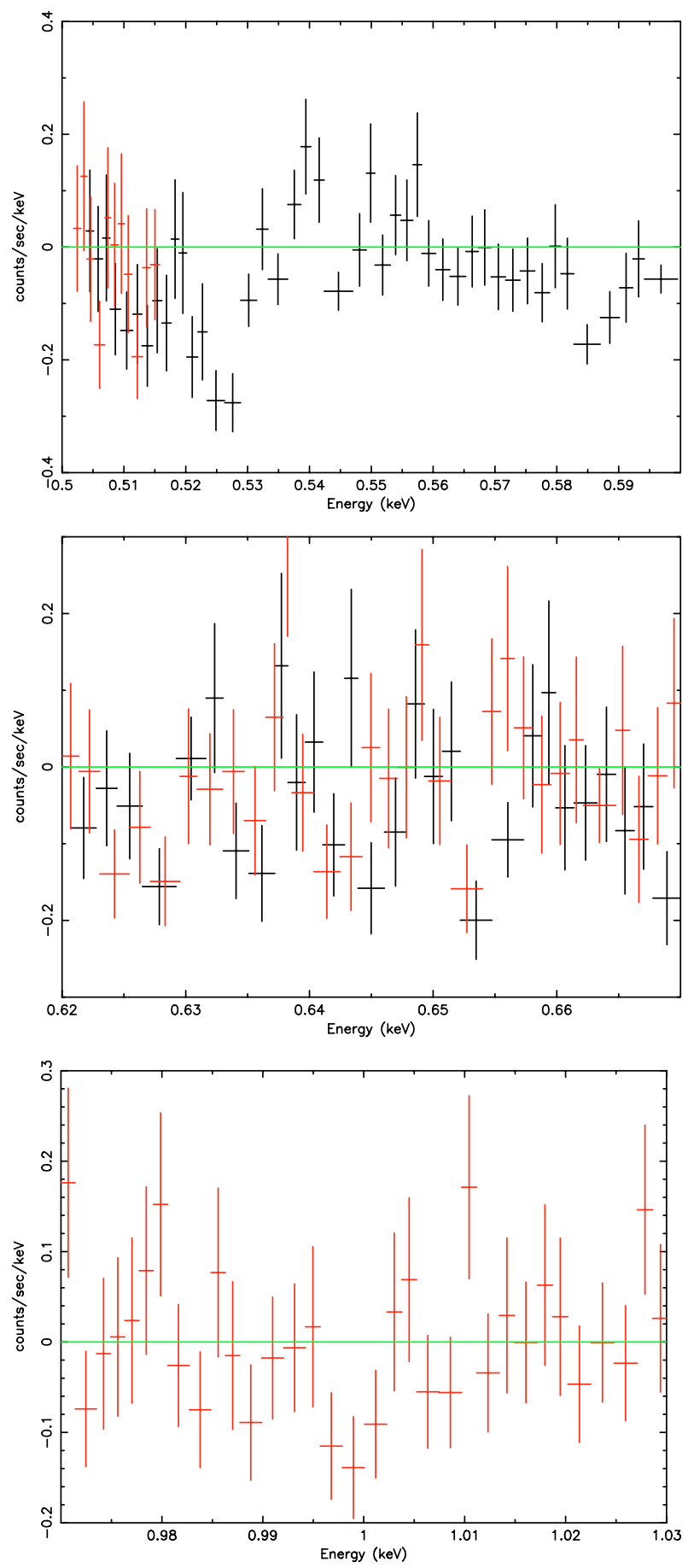

Fig. 6. Residuals for the RGS spectra, after fitting with the EPIC/p-n best fit model, in the energy ranges where the absorption lines reported in Table 2 are apparent.

the SAS documentation ${ }^{2}$. As we are interested in comparing the UV and X-ray fluxes, rather than in performing detailed fitting of the Spectral Energy Distribution, we did not deem it worthwhile to perform a more sophisticated analysis. After having checked that the fluxes for the three observations of each filter are consistent one another within the error, we obtained a

\footnotetext{
${ }^{2}$ http://xmm.vilspa.esa.es/sas/documentation/ watchout/uvflux.shtml
}

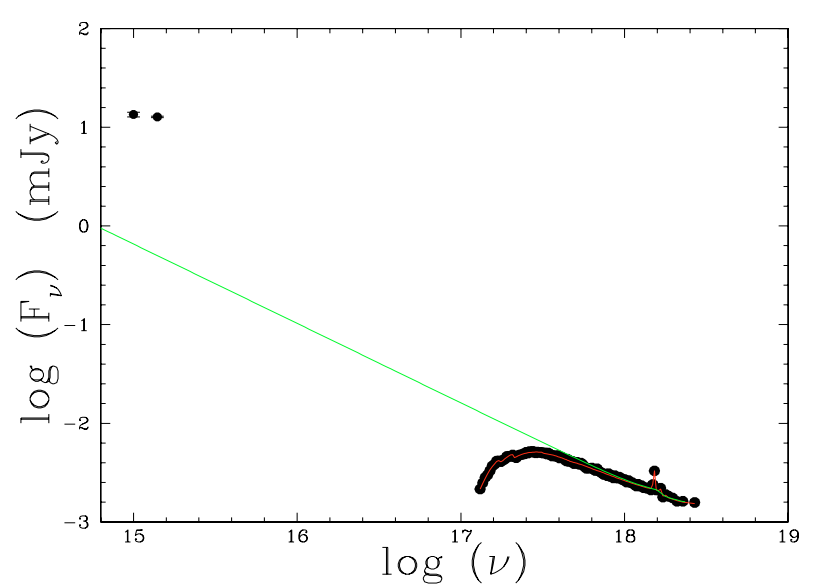

Fig. 7. UV fluxes as measured by the OM, along with the extrapolation of the best fit X-ray spectrum.

mean flux of $(7.33 \pm 0.04) \times 10^{-15} \mathrm{erg} \mathrm{cm}^{-2} \mathrm{~s}^{-1} \AA^{-1}$ in the $U V W 1$ filter and of $(3.52 \pm 0.06) \times 10^{-15} \mathrm{erg} \mathrm{cm}^{-2} \mathrm{~s}^{-1} \AA^{-1}$ in the $U V W 2$ filter. When corrected for extinction (after Cardelli et al. 1992), the fluxes are $(4.77 \pm 0.26) \times 10^{-14} \mathrm{erg} \mathrm{cm}^{-2} \mathrm{~s}^{-1} \AA^{-1}$ in the $U V W 1$ filter and $(4.48 \pm 0.15) \times 10^{-14} \mathrm{erg} \mathrm{cm}^{-2} \mathrm{~s}^{-1} \AA^{-1}$ in the $U V W 2$ filter. These fluxes are roughly in agreement with those found by Treves et al. (1990) with IUE on November 1983 (see their Fig. 8) in a quasi-simultaneous observation with EXOSAT, when the source had a X-ray flux very similar to that measured by XMM-Newton.

The UV fluxes are well above the extrapolation of the X-ray spectrum (see Fig. 7), indicating the clear presence of the UV bump. It may be tempting to take the lack of a soft excess, along with the presence of the UV bump, as an argument in favor of models of soft excesses which do not relate them with the accretion disc (e.g. Gierlinski \& Done 2004). It must be noted, however, that the large Galactic absorption makes the search for a soft excess in MCG+8-11-11 more difficult than usual.

\section{Discussion}

When compared to the October 1997 BeppoSAX observation (Perola et al. 2000), the flux of the source during the XMMNewton observation (April 2004) is about 20\% lower. The two spectra are, however, very similar; not only can the same model be successfully applied, but the spectral parameters (apart normalizations) are also the same within the errors. The major improvement provided by the XMM-Newton observation is that the width of the line is now much better constrained, ruling out any significant contribution from a relativistically broadened line.

The source was also observed by ASCA twice (Grandi et al. 1998), three days apart, on September 1995. There was also a coordinated OSSE/CGRO observation. The flux was about a factor 2 lower, but again the best fit parameters were consistent within the error with the ones we derived from the XMM-Newton data.

Therefore, despite rather large flux variations, there is no evidence of variations in the spectral parameters. In particular, 
the iron line flux is consistent with having remained constant, suggesting an origin in distant matter. The value of $R$, which in the same hypothesis should have been halved in the XMM-Newton observation, is so poorly determined by ASCA that no robust conclusions could be reached on this point.

MCG+8-11-11 is therefore another bright Seyfert 1 in which the presence of a strong relativistic line can be excluded with high confidence (see e.g. Matt et al. 2001; Pounds et al. 2003c; Bianchi et al. 2004, for other cases). The lack of such lines in many, if not most, local Seyfert galaxies is one of the more puzzling results of XMM-Newton. One possible explanation is ionization of the matter. For MCG+8-11-11, as discussed in Sect. 3.3, this solution seems unlikely.

Alternatively, the disc may be truncated, analogous with the Galactic black hole systems in hard state (e.g. Fender et al. 2004), even if such analogy seems not to hold as far as power spectral density is concerned (Uttley \& McHardy 2005). The lack of any measurable soft excess is certainly more easily explicable in the latter than in the former hypothesis. On the other hand, the UV excess suggests that a significant thermal disc emission is present. The flux density in the two filters diminishes slightly with frequency, suggesting we are in the region where the exponential rollover starts, i.e. $h v \sim k T_{\text {in }}$, where $T_{\text {in }}$ is the temperature of the inner radius of the disc. Assuming the value of the black hole mass given by Bian \& Zhao (2003), an $r^{-\frac{3}{4}}$ dependence of the disc temperature, and finally that the temperature in the innermost stable orbit in a Schwarzschild disc scales with the black hole mass as $T\left(6 r_{\mathrm{g}}\right) \sim 2 \times 10^{7}\left(M / M_{\odot}\right)^{-\frac{1}{4}} \mathrm{~K}$, we find an inner disc radius of about $75 r_{\mathrm{g}}$. Even if this number is different than the lower limit set by the iron line profile (e.g. $300 r_{\text {g }}$ ), given the large uncertainties in this very crude procedure, we can conclude that the UV flux is at least not inconsistent with the hypothesis of a truncated disc.

\section{Summary}

We have analyzed the XMM-Newton observation of the bright Seyfert 1 galaxy, MCG+8-11-11. The main results can be summarized as follows:

- The X-ray spectrum is well-fitted by a $\Gamma=1.8$ power law, plus a Compton reflection component and an $\mathrm{Fe} \mathrm{K} \alpha$ line. The spectrum is absorbed by warm material.

- The iron line is unresolved, and very likely originates in distant matter. The comparison of the line $E W$ to the amount of the Compton reflection component suggests iron underabundance.

- The warm absorber seems to be more complex than a single zone. Absorption lines are apparent in the RGS spectra, but their identification is ambiguous and, in any case, requires large matter velocities.

- No soft excess is required by the data. The large Galactic column density towards the source made, however, the search for such a component more difficult than usual.

- The UV fluxes measured by the OM are well above the extrapolation of the X-ray spectrum, indicating the presence of a UV bump.
Acknowledgements. G.M. and G.C.P. acknowledge financial support from MIUR under grant PRIN-03-02-23. This paper is based on observations obtained with XMM-Newton, an ESA science mission with instruments and contributions directly funded by ESA Member States and the USA (NASA).

\section{References}

Anders, E., \& Grevesse, N. 1989, Geo. Cosm. Acta, 53, 197

Antonucci, R. R. J. 1993, ARA\&A, 31, 473

Balestra, I., Bianchi, S., \& Matt, G. 2004, A\&A, 415, 437

Bian, W., \& Zhao, Y. 2003, MNRAS, 343, 164

Bianchi, S., Matt, G., Balestra, I., Guainazzi, M., \& Perola, G. C. 2004, A\&A, 422, 65

Bianchi, S., Miniutti, G., Fabian, A. C., \& Iwasawa, K. 2005, MNRAS, 360, 380

Cardelli, J. A., Sembach K. R., \& Mathis, J. S. 1992, AJ, 104, 1916

Chartas, G., Brandt, W. N., Gallagher, S. C., \& Garmire, G. P. 2002, ApJ, 579, 169

Dadina, M., Cappi, M., Malaguti, G., Ponti, G., \& De Rosa, A. 2005, A\&A, in press [arXiv: astro-ph/0506697]

Ehle, M., Breitfellner, M., Gonzalez-Riestra, R., et al. 2005, XMMNewton Users' Handbook, http://xmm.vilspa.esa.es/ external/xmm_user_support/documentation/uhb_2.1/

Elvis, M., Lockman, F. J., \& Wilkes, B. J. 1989, AJ, 97, 777

Fabian, A. C., Vaughan, S., Nandra, K., et al. 2002, MNRAS, 335, L1

Fender, R. P., Belloni, T., \& Gallo, E. 2004, MNRAS, 355, 1105

George, I. M., \& Fabian, A. C. 1991, MNRAS, 249, 352

Grandi, P., Haardt, F., Ghisellini, G., et al. 1998, ApJ, 498, 220

Hasinger, G., Schartel, N., \& Komossa, S. 2002, ApJ, 573, L77

Kaspi, S., Smith, P. S, Netzer, H., et al. 2000, ApJ, 533, 631

Kaspi, S., Brandt, W. N., George, I. M., et al. 2002, ApJ, 574, 643

Lubinski, P., \& Zdziarski, A. A 2001, MNRAS, 323, L37

Marconi, A., Risaliti, G., \& Gilli, R. 2004, MNRAS, 351, 169

Matt, G., Perola, G. C., \& Piro, L. 1991, A\&A, 247, 25

Matt, G., Fabian, A. C., \& Ross, R. R. 1996, MNRAS, 278, 1111

Matt, G., Fabian, A. C., \& Reynolds, C. S. 1997, MNRAS, 289, 175

Matt, G., Guainazzi, M., Perola, G. C., et al. 2001, A\&A, 377, L31

Matt, G. 2002, MNRAS, 337, 147

Matt, G., Guainazzi, M., \& Maiolino, R. 2003, MNRAS, 342, 422

Matt, G., Porquet, D., Bianchi, S., et al. 2005, A\&A, 435, 857

Molendi, S., Bianchi, S., \& Matt, G. 2003, MNRAS, 343, L1

Pecháček, T., Dovčiak, M., Karas, V., \& Matt, G. 2005, A\&A, 441, 855

Perola, G. C., Matt, G., Fiore, F., et al. 2000, A\&A, 358, 117

Piconcelli, E., Jimenez-Bailón, E., Guainazzi, M., et al. 2004, MNRAS, 351, 161

Pounds, K. A., Reeves, J. N., King, A. R., et al. 2003a, MNRAS, 345, 705

Pounds, K. A., King, A. R., Page, K. L., \& O’Brien, P. T. 2003b, MNRAS, 346, 1025

Pounds, K. A., Reeves, J. N., Page, K. L., et al. 2003c, MNRAS, 341, 953

Ravasio, M., Tagliaferri, G., Pollock, A. M. T., Ghisellini, G., \& Tavecchio, F. 2005, A\&A, 438, 481

Różańska, A., Czerny, B., Siemiginowska, A., Dumont, A.-M., \& Kawaguchi, T. 2004, ApJ, 600, 96

Strüder, L., Briel, U., Dennerl, K., et al. 2001, A\&A, 365, L18

Treves, A., Bonelli, G., Chiappetti, L., et al. 1990, ApJ, 359, 98

Turner, M. J. L., Abbey, A., Arnaud, M., et al. 2001, A\&A, 365, L27

Turner, T. J., Mushotzky, R. F., Yaqoob, T., et al. 2002, ApJ, 574, L123

Uttley, P., \& McHardy, I. M. 2005, MNRAS, in press [arXiv: astro-ph/0508058]

Wilms, J., Reynolds, C. S., Begelman, M. C., et al. 2001, MNRAS, 328, L27 